\author{
Universidad Santo Tomás
}

Facultad de Administración de Empresas

\title{
AUDI MÉXICO UN MODELO A SEGUIR EN INNOVACIÓN TECNOLÓGICA PARA LA CAPACITACIÓN EN COLOMBIA
}

\author{
Trabajo de Opción de grado \\ Periplo Internacional México
}

\author{
Presentado por: \\ Juan David Sabogal Castro \\ Michael Steven Orozco Romero
}

Tutores:

Norha Castillo Castro

Mauricio Novoa Campos

Bogotá D.C. 15 de Enero del 2019 


\section{Tabla de contenido}

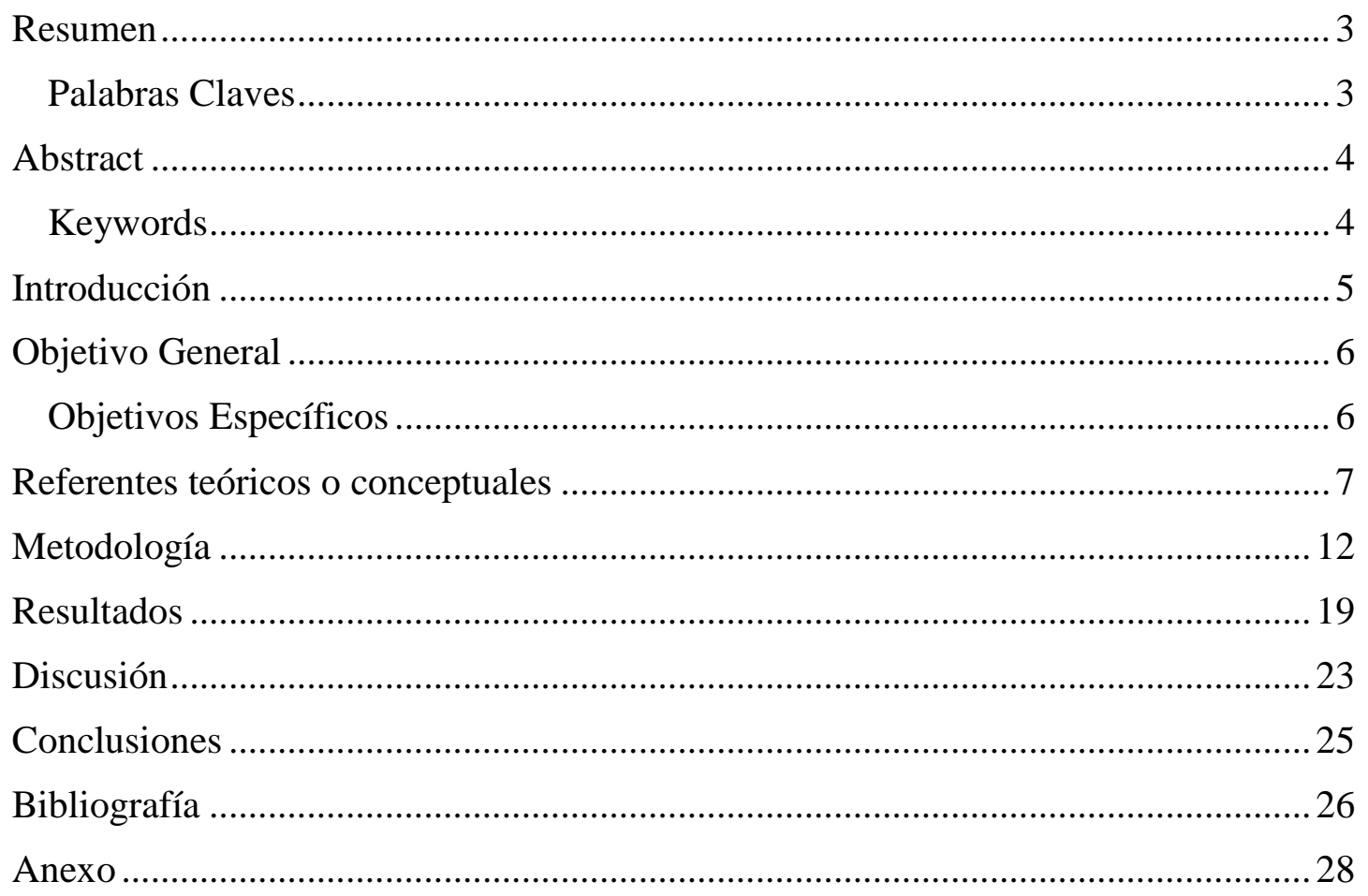




\section{Resumen}

La finalidad de este informe es evidenciar las potencialidades y beneficios que otorga las capacitaciones empresariales dentro de las organizaciones y la innovación tecnológica para la adaptación al mercado laboral, con el fin de obtener una idea más clara acerca de la importancia que le dan las empresas a la capacitación al pasar de los años, tomando como referencia puntos de vista de diferentes autores y estadísticas de las respectivas investigaciones que permiten analizar el estado actual de las empresas colombianas en cuanto al tema de capacitaciones empresariales de calidad, conociendo principales obstáculos, problemas, sectores con mayor porcentaje de implementación y beneficios de esta.

Se tomó como punto de partida la capacitación empresarial de Audi México y como dicha empresa tiene dentro de sus planes y presupuesto el desarrollo de una capacitación enfocada a la calidad y a la perfección del proceso-producto, para que de esta manera se puedan satisfacer y cumplir con las necesidades y expectativas del mercado objetivo.

\section{Palabras Claves}

Capacitación, tecnología, empresa, innovación, colaboradores, obstáculos, beneficios, calidad, educación. 


\begin{abstract}
The purpose of this report is to highlight the potential and benefits of business training within organizations and their constant innovation to adapt to the labor market, in order to get a clearer idea about the importance that companies give to the training over the years taking as a reference points of view of different authors and statistics of the respective investigations that allow us to analyze the current state of the Colombian companies regarding the topic of quality business training, knowing the main obstacles, problems, sectors with greater percentage of implementation and benefits of this.
\end{abstract}

The business training of Audi Mexico was taken as a reference and as such company has within its plans and budget the development of a training focused on the quality and perfection of the process-product, so that in this way they can satisfy and comply with the needs and expectations of the target market.

\title{
Keywords
}

Business Training, technology, company, innovation, employees, obstacles, benefits, quality, education. 


\section{Introducción}

El centro de especialización de Audi en México es un modelo claro de innovación tecnológica en la formación de empleados, como lo declaró el gerente general: "Procuramos crear para nuestros empleados condiciones de trabajo atractivas en todos y cada uno de los centros en todo el mundo. En el nuevo Centro de Formación ofrecemos las mejores posibilidades de capacitación para todos los colegas y, con ello, situamos a las personas y su desarrollo personal en primer plano" (Peter Mosch). En Audi no solo se piensa en el beneficio de la compañía también se tiene en cuenta la evolución de cada colaborador dentro de la misma, de allí el motivo del presente informe.

Partiendo del hecho de que para las organizaciones el recurso más importante es el talento humano es pertinente saber qué están haciendo las empresas para potencializar las capacidades de cada colaborador, es ahí donde la capacitación entra a desarrollar un papel fundamental dentro de los procesos en las compañías y más aún en un contexto globalizado donde hay que adaptarse a cambios tecnológicos, económicos, políticos y sociales para poder sobrevivir en un mercado altamente competitivo, el personal debe estar preparado para enfrentarse a dichas situaciones y poder desempeñarse de manera correcta.

No es suficiente que las organizaciones realicen una capacitación para cumplir un requisito, sino del efecto esperado medido en el alcance de nuevos conocimientos, 
desarrollo de nuevas habilidades, mejoramiento personal, calidad del trabajo y aumento de la productividad que cada empleado adquiera para el progreso personal de cada uno y para el alcance de los objetivos empresariales. En Colombia 8 de cada 10 empresas capacitan a sus empleados según un estudio realizado por la Asociación Nacional de Empresarios de Colombia, las preguntas que surgen son ¿Tienen un verdadero impacto?, ¿Qué limitaciones hay?, ¿Existe innovación tecnológica? En el contenido del informe se trata de dar respuesta a dichas incógnitas.

\section{Objetivo General}

Presentar como referencia el centro de especialización de Audi en México para saber el estado de Colombia respecto a la capacitación.

\section{Objetivos Específicos}

- Describir de manera general el centro de especialización de Audi en México y su modelo de funcionamiento.

- Identificar si en Colombia existe innovación tecnológica en los procesos de capacitación y si hay un modelo similar como el de Audi en México.

- Analizar los principales beneficios y obstáculos que hay en los programas de capacitación en Colombia. 


\section{Referentes teóricos o conceptuales}

Al realizar el proceso migratorio hacia México y después de hacer las respectivas visitas programadas en la agenda, se coincidió que la visita que más llamó la atención fue la de Audi ubicada en Puebla, en especial el centro de especialización para la capacitación del personal, por la gran innovación tecnológica que en ella se pudo observar. Es por ello que se escogió como tema la innovación tecnológica en la capacitación, tomando como referencia el centro de especialización en Audi.

Para hablar de innovación tecnológica enfocada en los procesos de capacitación en las empresas, es necesario desglosar y definir dichos conceptos para una mayor comprensión.

Innovación

La innovación es un término que puede interpretarse de diferentes maneras; para muchas personas la innovación es la creación de un nuevo producto en el mercado y lo relacionan directamente con algo material algo tangible, otros piensan que la innovación hace parte de un producto, servicio o proceso que aunque ya existe se realiza de una forma diferente creando valor.

Los siguientes autores definen innovación así:

"la introducción de nuevos bienes y servicios en el mercado, el surgimiento de nuevos métodos de producción y transporte, la consecución de la apertura de un nuevo mercado, la generación de una nueva fuente de ofertas de materias primas y el cambio en la organización en su proceso de gestión” (Schumpeter, 1942). 
"la búsqueda y el descubrimiento, la experimentación y adopción de nuevos productos, nuevos procesos de producción y nuevas formas organizacionales" (Dosi, 1988).

“es la introducción de un nuevo, o significativamente mejorado producto (bien o servicio), de un proceso, de un nuevo método de comercialización o de un nuevo método organizativo al mercado y la sociedad" (Manual de Oslo, 2005).

Innovación tecnológica

La tecnología ha sido un elemento esencial para la misma innovación, pues conlleva ciertos conocimientos y técnicas que permiten transformar ya sea un elemento o entorno con la finalidad de suplir una necesidad. Así se define:

“La tecnología es una ciencia que aplica de forma organizada el conocimiento científico a tareas practicas o a la resolución de problemas específicos. Para ello utiliza teorías y métodos científicos de otros saberes, adaptándolos para obtener determinados fines. Asimismo, la tecnología como ciencia, responde a las necesidades de la sociedad y tiene como propósito contribuir a mejorar la calidad de vida, no solo a nivel de necesidades físicas sino también de expectativas o deseos"(Cervera, 2010, p 118).

En cuanto a la innovación tecnológica "surge tras la utilización de la tecnología como medio para introducir un cambio en la empresa. Este tipo de innovación tradicionalmente se ha venido asociando a cambios en los aspectos más directamente relacionados con los medios de producción. El único agente imprescindible para que 
exista innovación tecnológica es la misma empresa ya que es la responsable de su utilización para introducir el cambio" (Fundación COTEC, 2001)

También es definida como:

"la primera aplicación de la ciencia y la tecnología en una nueva dirección, seguida de un éxito comercial” (OCDE, 1971, p 11).

Esta definición involucra directamente a la I+D (Investigación y desarrollo) que debe estar presente en toda organización que quiera ser competitiva en el mercado ya que cumple una función importante al ser la base de la innovación tecnológica.

\section{Capacitación}

Se considera que la capacitación es la etapa donde los empleados de una organización adquieren conocimientos específicos para cumplir con una tarea, función o actividad determinada.

Según Chiavenato "La capacitación es un medio que desarrolla las competencias de las personas para que puedan ser más productivas, creativas e innovadoras, a efecto de que contribuyan mejor a los objetivos organizacionales y se vuelvan cada vez más valiosas"(Chiavenato,2009, p 371)

Existen diferentes teorías que aunque no hablen directamente acerca de la capacitación, influyen en sus procesos e implementación. Como lo propone Deming (1989) con los 14 principios de calidad que conducen a la productividad ya que se cometen menos errores; por otro lado Ouchi (1982) manifiesta que la cultura de una organización se enriquece a través de la administración participativa que a la vez 
puede conducir al desarrollo de las habilidades y destrezas del personal en el puesto de trabajo. Otro factor determinante es la motivación y retención del personal para el despliegue de sus habilidades y destrezas en beneficio propio y de la organización. Respecto a lo anterior se evidencia que el rendimiento del colaborador está condicionado, no solo por aspectos como el procedimiento, el sistema de trabajo utilizado o la tecnología disponible, sino también por factores motivadores y se relacionan con el enriquecimiento del puesto de trabajo.

Es importante mencionar el impacto que tiene hoy en día la capacitación en las empresas como se define a continuación. "la capacitación actualmente se relaciona ampliamente con la educación a diferencia de algunos años que se consideraba un gasto a fondo perdido o un trámite para cumplir con requisitos u obligaciones enmarcadas en las leyes laborales" (A. Orozco, p 4, 2017).

Teniendo en cuenta el punto de vista anterior es precisamente lo que está haciendo Audi a través de su centro de especialización, pues su objetivo es formar profesionales en todo su personal con los más altos estándares de calidad, mediante la innovación tecnológica en cada uno de sus procesos cumpliendo así con las metas tanto de los colaboradores como de la organización.

Modelo Tripe - Hélice

Implementado por Audi, tiene como principal componente la vinculación entre la academia, el gobierno y la empresa con el fin de capacitar a los aprendices para que puedan desempeñarse correctamente en un mercado laboral. 
"La triple Hélice es una expansión del papel del conocimiento en la sociedad y de la universidad en la economía. La Universidad está experimentando una transformación dual: una expansión de las misiones a fin de incluir el desarrollo económico y social, así como la formación, la reproducción cultural y la investigación y el cambio de un individuo a un foco de organización en cada misión.” (Henry Etzkowizt 2002)

"El papel de intercambio de conocimientos y la cooperación en investigación entre la investigación pública y el sector empresarial ha recibido una atención creciente en el análisis de la innovación y el cambio tecnológico.” (Doris Schartinger 2002)

Papel de cada factor del modelo

Universidad:

Juega un papel muy importante en las actividades socioeconómicas de un país, en donde se ve reflejado que la universidad además de desarrollar su actividad propia de enseñanza-aprendizaje, participe en la creación de nuevas empresas e incentive el emprendimiento, de este modo la universidad se puede convertir en un punto de partida para que dichas nuevas organizaciones se puedan formar. Los mecanismos de cooperación en cuanto a la investigación, las empresas tienen la posibilidad de facilitar recursos a la universidad con el propósito de equipar e impulsar proyectos a través de donaciones o becas. 
Gobierno:

El papel del gobierno es un factor primordial para el desarrollo del Modelo TripleHélice debido a que se necesita una participación activa del gobierno a través de la legislación, incentivos y mecanismos fiscales adecuados para el desarrollo y dinamismo de las relaciones que surgen entre la empresa y la universidad.

Empresa:

Su papel importante es el desarrollo tecnológico, debido a que los cambios constantes en la tecnología propicia un ambiente de generación de nuevos conocimientos y de esta manera estimule el crecimiento en las empresas mediante mecanismos de cooperación con las universidades; involucrándose en la formación de capacidades que apoyen a las empresas por medio de las universidades a implantar nuevas formas de creación de fuentes de innovación para el desarrollo de altas tecnologías en las empresas.

\section{Metodología}

Al evidenciar la gran importancia que se le da a la capacitación del personal en Audi y la apuesta por una innovación tecnológica de la magnitud del centro de especialización que poseen, surgió la inquietud de saber que tan lejos está Colombia en esta área. A continuación se va a desarrollar un respectivo análisis tomando como referente el centro de especialización y posteriormente saber cómo se encuentra Colombia en este ámbito. 
Centro de Especialización - Audi México

Ubicado en San José Chiapa, Puebla con un espacio de $20.000 \mathrm{~m} 2$ siendo el complejo más grande y moderno de américa latina para la formación del personal, fue creado con la intención de capacitar con los estándares más altos de calidad a todo su personal, ofreciendo alrededor de 1.500 cursos anuales a sus empleados y becarios, con más de 450.000 horas de capacitación por año, y hasta el año 2016 más de 2.800 colaboradores han pasado por los cursos de capacitación. Además en este centro se realiza formación profesional dual que consiste en la combinación empleo formación haciendo que el aprendiz adquiera conocimientos en el centro educativo y la empresa complemente dichos conocimientos llevándolos la práctica. Así lo describe Thomas Sigi responsable de RR.HH.

"una capacitación de primera clase, posibilita que cada uno de los audianos esté listo para el inicio de la producción. Como empleador atractivo, queremos formar a nuestros colaboradores mexicanos de forma individual atendiendo a sus necesidades y al mismo tiempo velar por implantar los máximos estándares en el sector a escala global. De esta forma, estamos brindando a nuestro equipo y a Audi México una preparación a la medida para el futuro". 
Modelo Triple - Hélice en Audi

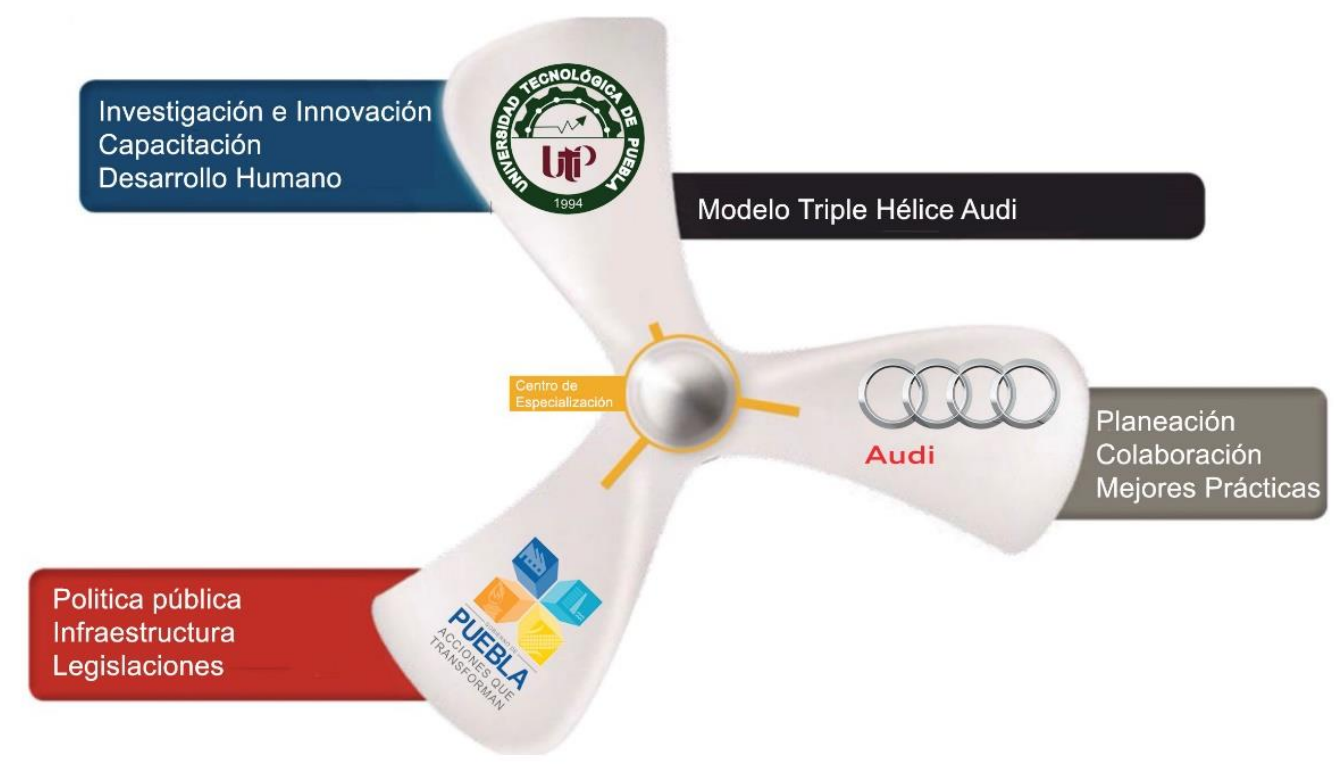

\section{Elaboración propia.}

Es administrado y operado por la Universidad Tecnológica de puebla bajo la consejería de la empresa Audi y el Gobierno de puebla, el centro nace con la visión de formar una nueva generación de profesionales de la industria automotriz dotándolos de las competencias necesarias para que al final de su capacitación se encuentren en capacidad de incursionar en el mercado laboral del sector automotriz, el centro cuenta con equipamiento de última generación y recursos pedagógicos de educación dual donde el $80 \%$ es la práctica y el $20 \%$ la teoría, con lo que se garantiza que sus egresados obtengan un nivel de preparación de excelencia tomando en consideración los más altos estándares de calidad internacional.

Ofreciendo programas de certificación en mecatrónica, tecnología del vehículo, mecánico de carrocerías, herramientas y procesos de producción, de igual manera 
ofrece capacitación de primera clase a cargo de instructores entrenados en Alemania para ofrecer diversos conocimientos expertos con la máxima precisión en los detalles, para que de esta manera se asegure la calidad y la sustentabilidad en sus procesos.

La alianza estratégica entre Audi y el gobierno estatal de Puebla consiste en hacer una fuerte apuesta al futuro, el gobernador asumió el compromiso personal y político de transformar a puebla y devolverle el lugar que le corresponde entre los estados del país y ante el mundo, así que uno de los principales objetivos que se fijaron fue recuperar la confianza de los inversionistas y convertir al estado en uno de los principales en el sector automotriz a nivel nacional y global, la visión de progreso y desarrollo que se ofreció para puebla se está materializando, una clara muestra de ello es la inversión extranjera directa, La empresa Audi eligió a José Chiapas para instalar una nueva planta armadora de autos con una inversión inicial de 1.300 millones de dólares, gracias a ello se han creado 10.000 empleos mientras que en su fase de operación para los próximos años se generaran 16.000 empleos permanentes y se producirán 150.000 vehículos al año, multiplicando las oportunidades de progreso y bienestar de la población.

Innovación tecnológica en los programas de capacitación en Colombia Existe una brecha importante en la innovación en Colombia pues como lo menciona Sofía González en su publicación la innovación como fuente de desarrollo se debe entre otras cosas por los problemas de desigualdad y desarrollo que hay en el país, además del bajo nivel de inversión que se le da a puntos claves como actividades científicas, tecnológicas e innovadoras comparada con otros países de la región como 
Chile, Costa rica y México quienes ocupan los primero lugares de Latinoamérica según el índice mundial de innovación 2017. Por otro lado el factor humano es determinante a la hora de aportar capacidades para innovar generando nuevos conocimientos adaptándose a los nuevos contextos en un entorno global, también son necesarias sus habilidades técnicas y empresariales que faciliten la transferencia y difusión de tecnología.

Según Javier Echeverri Hincapié, Country Manager de Manpowergroup Colombia “En el entorno laboral Colombiano hay que empezar a ver la capacitación como un tema clave para las empresas, sobre todo por el cambio en el mercado laboral que es exigido en un contexto donde la tecnología ha transformado todas las dinámicas de las compañías"

El costo de implementar tecnología aplicada a la formación del personal varía según el modelo de negocio, no obstante para la mayoría de las empresas en Colombia la capacitación es subestimada. La encuesta nacional de inteligencia de negocios ejecutada por la Asociación Colombiana de Ingenieros de Sistemas reveló que solo el 4\% de la inversión en tecnología de las organizaciones participantes en la encuesta se aplica a iniciativas de formación. Omar Villota presidente de la Asociación de Inteligencia colectiva Iberoamericana señala que la inversión en tecnología aplicada a programas de capacitación en Colombia es mínima. "las empresas dejan solo a sus trabajadores para asumir la capacitación y actualización que la organización requiere". 
Con respecto a las empresas que están invirtiendo para capacitar a sus colaboradores estas se encuentran en dos grupos: las que están ingresando al mundo del E-learning y las que implementan universidad corporativa. El primer grupo inician con proyectos en servicio de consultoría, virtualización de contenidos y plataformas virtuales mientras que el segundo grupo implementan los planes de carrera, los esquemas de compensación y la administración de competencias.

En Colombia las principales razones por las cuales las empresas no tienen como objetivo primordial el desarrollo de un excelente programa de capacitación y formación de personal son la falta de presupuesto (24\%) y la disminución de gastos por bajas utilidades o pérdidas (16\%).

Algunos desafíos u obstáculos evidenciados en el desarrollo de programas de capacitaciones son los siguientes: La poca disponibilidad y falta de tiempo por parte de los formadores o funcionarios $(37 \%)$, el presupuesto limitado $(28,9 \%)$ y la ausencia de programas específicos o especializados $(7,7 \%)$ y en menor impacto, los gastos y costos de los programas $(2,8)$ y finalmente la alta rotación de personal $(2,1 \%)$. De manera que si en Colombia las empresas no invierten en capacitación mucho menos invierten en innovación tecnológica aplicada a los procesos de capacitación.

Tomando como referencia el modelo Triple- Hélice evidenciado en Audi, En Colombia el SENA es una entidad fundamental para llevar a cabo este modelo ya que la institución está facultada por el Gobierno con un vínculo estratégico con el sector 
empresarial en todo el territorio nacional, una parte importante para su desarrollo es el contrato de Aprendizaje que implementan.

Uno de los grandes propósitos del SENA, en cabeza de su directora, ha sido promover entre los empresarios el Contrato de Aprendizaje como una fórmula ganadora para la entidad y sobre todo para sus aprendices, la cual permite que los empresarios accedan al talento humano con pertinencia y de calidad al ser Coformadores, así como brinda a los aprendices la oportunidad de realizar las prácticas empresariales en ambientes reales, facilitando su inserción laboral formal.

Durante 2016, más de 345.000 aprendices tuvieron Contrato de Aprendizaje, componente fundamental en el desarrollo de su proceso formativo lo cual permitió alcanzar un cumplimiento del 100\% frente a la meta establecida. A julio de 2017, 264.147 aprendices han accedido a este beneficio. El 2016 terminó con 30.917 empresas reguladas logrando espacios de práctica. El esfuerzo realizado facilitó convencer a 15.306 empresas de contratar 62.280 aprendices voluntarios. Para la vigencia 2017 se gestionan 407.000 espacios de práctica. Al mes de julio se habían conseguido 264.147 contratos de aprendizaje, de los cuales, 50.354 son contratos voluntarios.

El director de Empleo, Trabajo y Emprendimiento, Gabriel Giraldo destacó la labor del SENA para promover entre los empresarios el Contrato de Aprendizaje, "el Contrato de Aprendizaje que nos permite romper con ese paradigma de que el empresario no contrata porque no se tiene experiencia. Es un beneficio para el 
aprendiz en la medida en que se forma en escenarios reales y para la empresa porque forma recursos a su medida".

\section{Resultados}

- Capacitación del personal Total Nacional

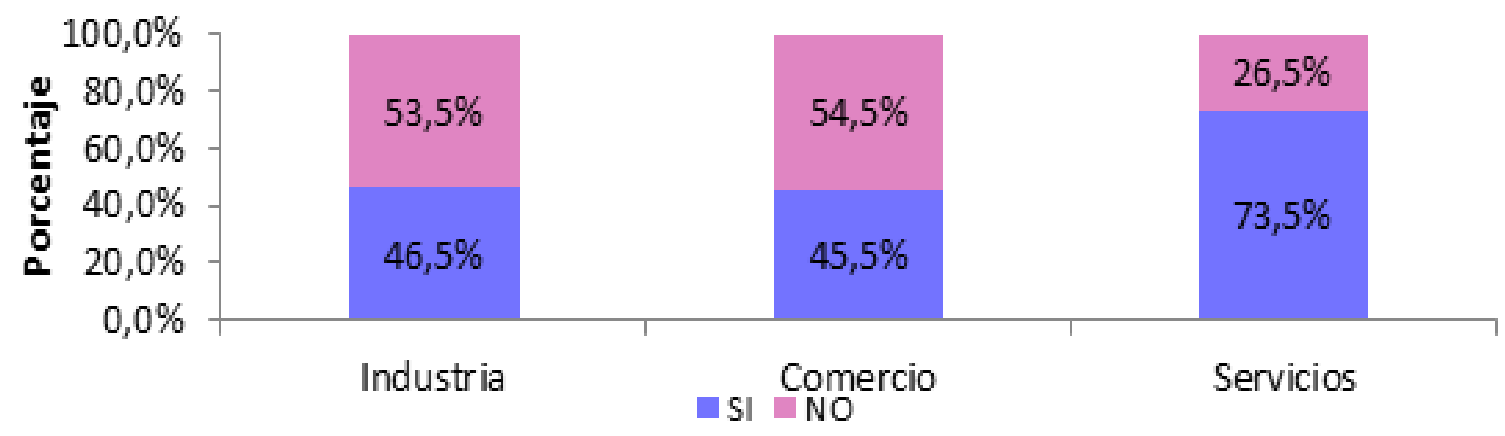

Gráfica 1.

Fuente: DANE-EFCH

- Razones para establecer capacitación

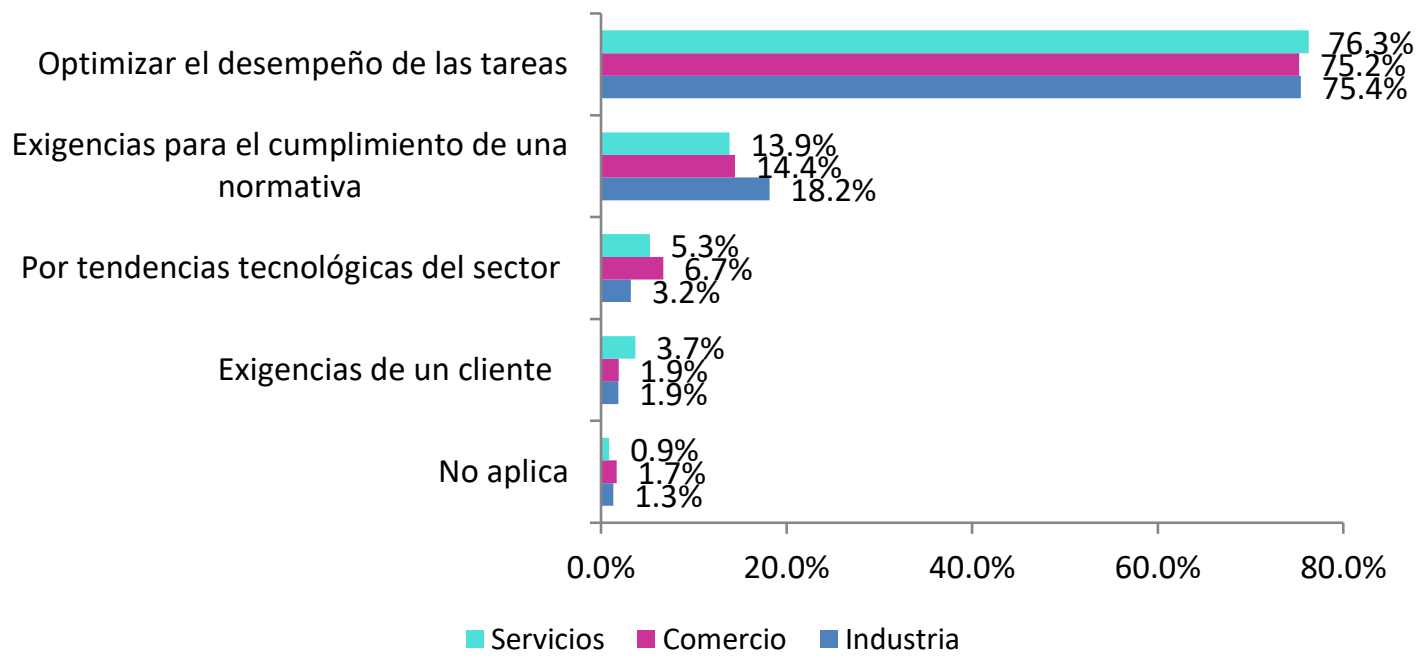

Grafica 2

Fuente: DANE 
- Aspectos mejorados con la capacitación Total Nacional

Optimizacion de los procesos de producción o prestación de servicios.

Mejora de la calidad del producto o servicio.

Optimizacion de los procesos administrativos.

Optimización de los insumos.

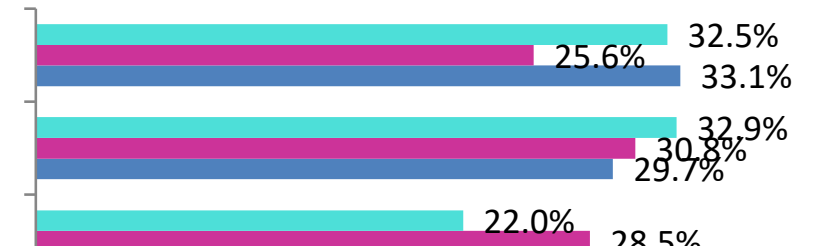

$28.5 \%$

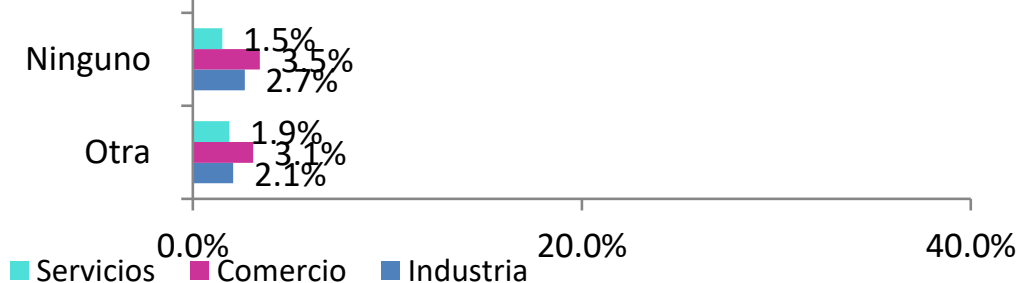

\section{Gráfica 3.}

Fuente: DANE-EFCH

- Entidades Certificadoras

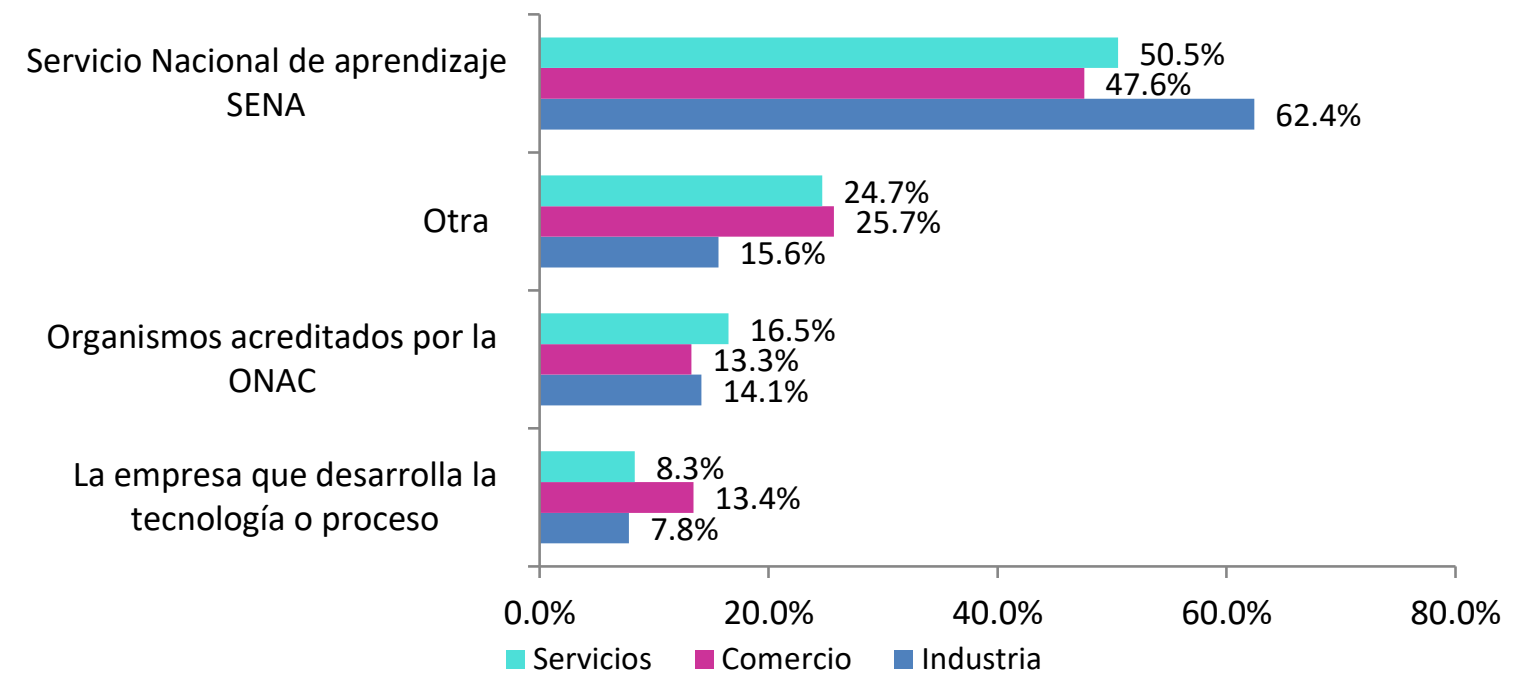

\section{Grafica 4}

Fuente: DANE 
- Formación profesional integral - Febrero 2016

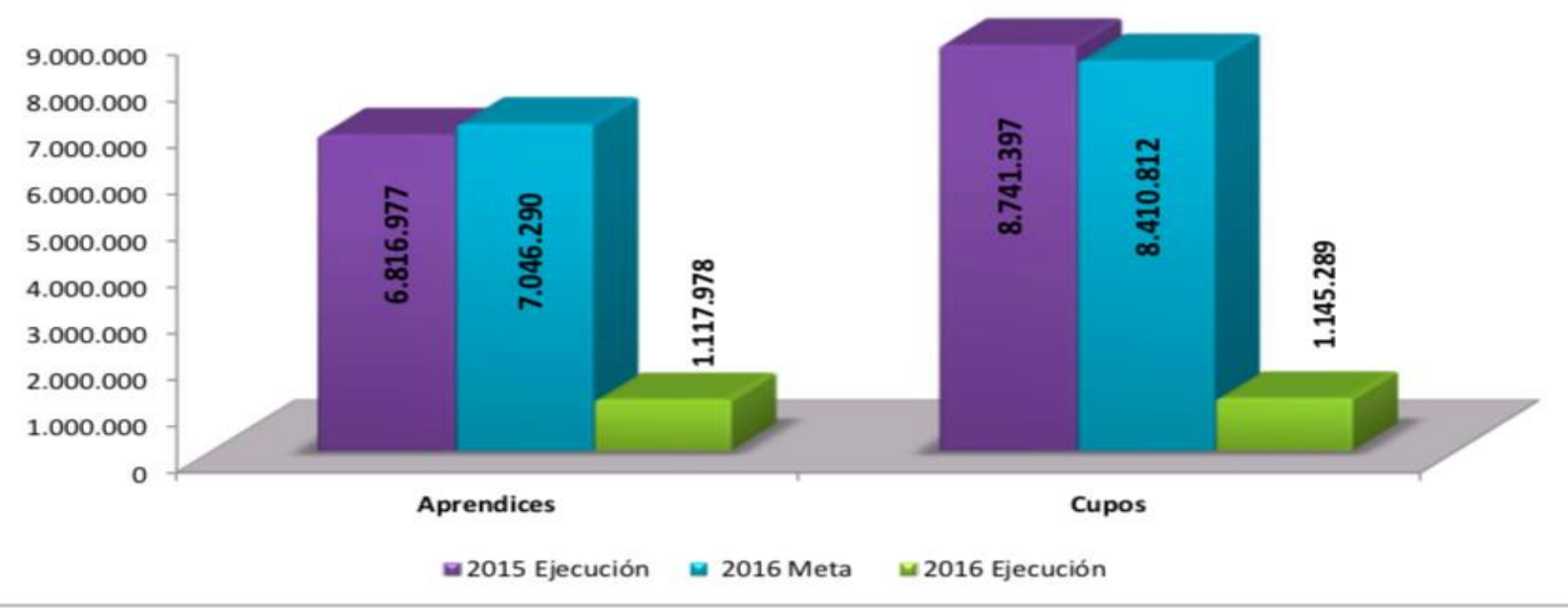

Notas:

Incluye Centros de Formación, Convenios Ampliación de Cobertura

Cupos: $\quad$ La cifra de cupos se establece contabilizando un cupo por cada curso o programa de formación que realice el alumno

Aprendices: $\quad$ La cifra de aprendices para Formación Titulada, se establece contabilizando un aprendiz por cada curso o programa de formación

que realice el alumno. Para Formación Complementaria, se establece contabilizando una sola vez el aprendiz, independientemente del número de cursos o programas de formación que realice

Fuente: Dirección de Planeación y Direccionamiento Corporativo, Grupo de Gestión de la Información y Evaluación de Resultados Aplicativo Sofía Plus

\section{Grafica 5}

\section{Fuente: SENA}

- Contrato de Aprendizaje - Febrero del 2016

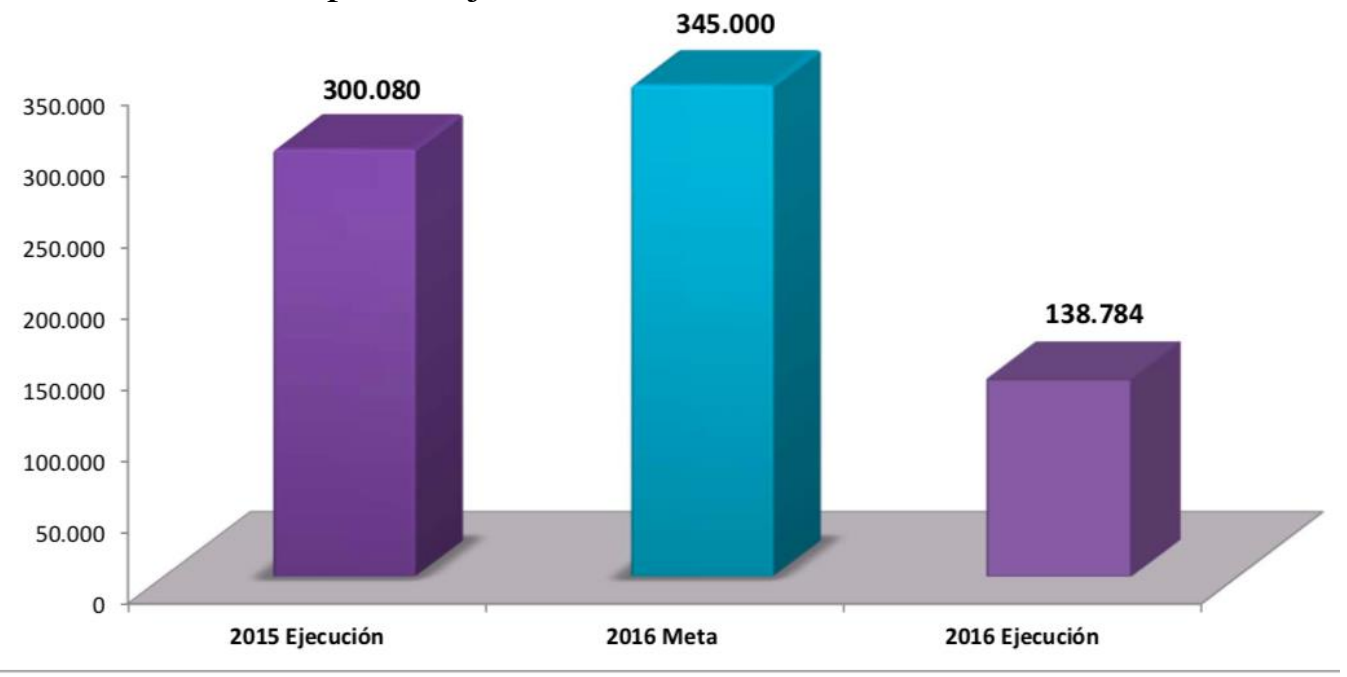

Fuente: Dirección Empleo y Trabajo - Grupo de Agencia Pública de Empleo - Aplicativo Sistema de Gestión Virtual de Aprendices SGVA

\section{Grafica 6}

Fuente: SENA 
- Motivos de No Capacitación Total Nacional

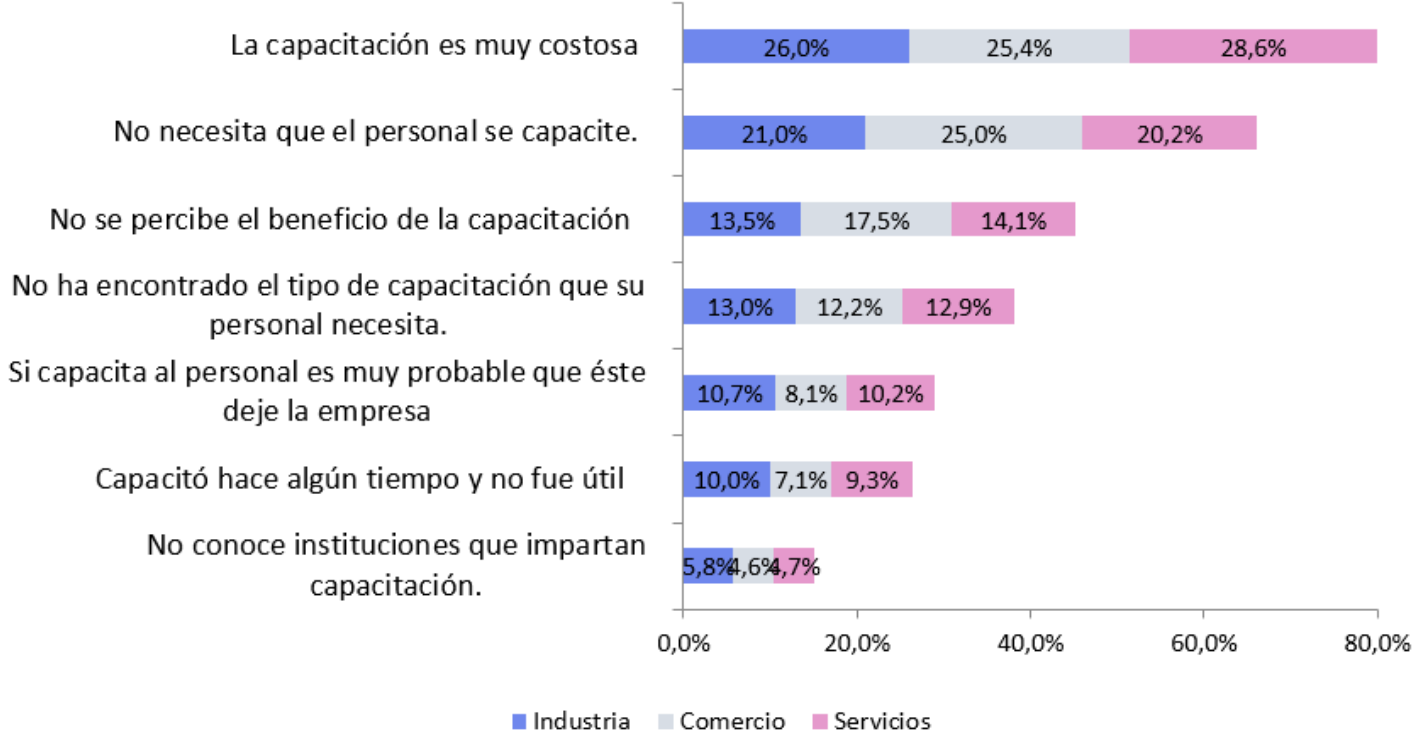

Gráfica 7.

Fuente: DANE - EFCH

- Obstáculos de la capacitación

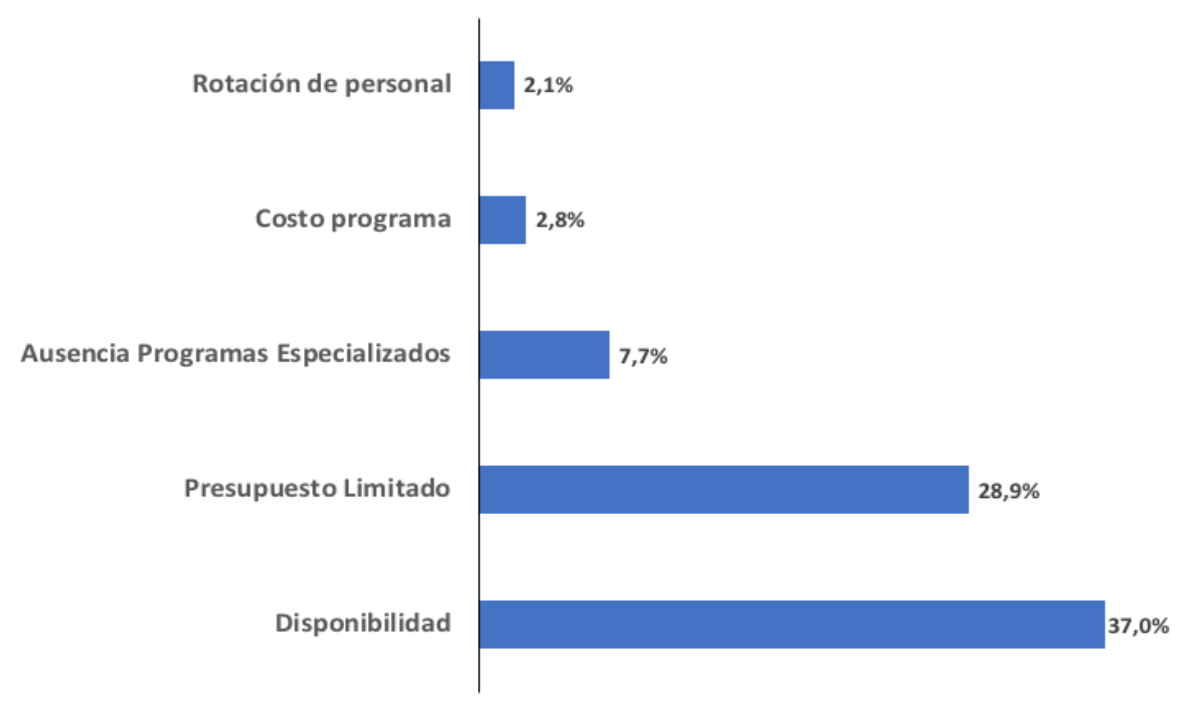

Grafica 8.

Fuente: Elaboración propia 


\section{Discusión}

Las empresas del sector de servicio en Colombia son las que más implementan programas de capacitación, una razón es el alto nivel de especialización que se necesita en su rama y la diversidad de tareas que se deben desarrollar.

Llama la atención que dentro de las razones para establecer la capacitación, el porcentaje de las exigencias para el cumplimiento de una normativa sea superior a las exigencias de un cliente, pues la razón de ser de cada empresa es precisamente este por lo cual se debe hacer todo lo posible para satisfacer sus respectivas exigencias.

Si se hace una buena ejecución en la capacitación se pueden obtener mejoras importantes como la optimización de los procesos de producción o prestación de servicios, mejora la calidad del producto o servicio y la optimización de procesos administrativos.

Hay que resaltar la importancia del Servicio Nacional de Aprendizaje SENA debido a que es la entidad certificadora en Colombia que tiene el porcentaje más alto en capacitación, formando técnicos y tecnólogos capaces de responder a los requerimientos de los sectores empresariales en el mercado laboral.

Dentro de los argumentos de las empresas para no realizar programas de capacitación se destacan principalmente el costo, la no necesidad de implementarse, la percepción de que no causan ningún beneficio. 
Generalmente lo que buscan las compañías es optimizar procesos, es por ello que la tendencia más viable es la virtualización de procesos como por ejemplo E-Learning, que dentro del área de recursos humanos enfocado a la capacitación es un beneficio notable para el área, debido a que principalmente se optimiza tiempo, recursos (papel) y para el colaborador es mucho más fácil este método de aprendizaje, con un proceso de acompañamiento y seguimiento del formador se garantiza una correcta capacitación, que en un futuro es beneficio para la compañía debido a que se mantiene un personal con habilidades potenciadas y que genera una optimización de procesos que se puede ver reflejada en los KPI's al momento de evaluar el desempeño del colaborador antes de tener la capacitación y después de esta.

Es por ello que actualmente las empresas deben tener más conciencia acerca de que la capacitación es un Plus frente a la competencia, y que debido a eso deben contar con un plan o programa de capacitación especializado para mejorar distintas variables dentro de la compañía ya sean: Optimización del desempeño dentro del cargo, exigencias para el cumplimiento de las normativas, tendencias del sector, o exigencias del cliente, si las empresas empiezan a tener dentro de sus costosbeneficios un presupuesto para la formación de sus colaboradores en un plazo mediano-largo se pueden ver reflejadas el retorno positivo de dicho costo. 


\section{Conclusiones}

Las empresas en Colombia sobre el papel tienen dentro de sus proyectos un programa de capacitación para sus empleados pero no se ve reflejado en el impacto que estas tienen para la compañía, además no existe la suficiente consciencia de darle la merecida relevancia a que cuenten con un plan de presupuestos para la innovación tecnológica aplicada a las capacitaciones en pro de la calidad del servicio o producto acorde con las exigencias del constante cambio del mercado laboral.

Se recomienda especialmente al área del talento humano de las empresas en Colombia por ser el departamento encargado de la gestión directa del personal de la compañía y su respectivo bienestar dentro de la organización, que consigan e incentiven a los directivos de las empresas para que dentro de su planes de dirección tengan en cuenta la capacitación y sus respectivos procesos como eje fundamental de su modelo de gestión, ya que una de las principales razones por la cual una organización se mantiene en el mercado es a través de la formación, exactamente igual a como una sociedad crece y un país se desarrolla.

Acoplándose a la actualidad se entiende que el activo más valioso para una compañía son sus colaboradores, es por ello que si las empresas cuentan con un programa de capacitación específico y de calidad se podrán potenciar las competencias de dichos empleados para generar una ventaja competitiva sostenible en el tiempo y de esta manera se pueda cumplir con los objetivos de la empresa. 


\section{Bibliografía}

America Learning Media. (2010). Capacitación + Tecnología: Cuánto y en qué invierten las organizaciones. Obtenido de http://www.americalearningmedia.com/edicion012/141-tendencias/1182-capacitacion-tecnologia-cuanto-y-en-que-invierten-lasorganizaciones

Angel, B. (s.f.). El Concepto de Innovacion . Obtenido de https://www.ceipa.edu.co/lupa/index.php/lupa/article/view/94/182

Audi. (2014). Audi Mexico: Inaugurado nuevo Centro de Especializacion. Obtenido de Audi Mexico: http://www.audi.com.mx/mx/web/es/audi-en-mexico/mediacenter/noticias/141021_audi-mexico--inaugurado-nuevo-centro-deespecializacion.html

Blasco, L. (2017). ¿Cuáles son los países más y menos innovadores de America Latina? Obtenido de BBC - Mundo: https://www.bbc.com/mundo/noticias-40293495

Cervera, D. (2010). Tecnología: investigación, innovación y buenas prácticas. Ministerio de Educación de España.

Chang, H. (2010). El modelo de la triple hélice como un medio para la vinculación entre la Universidad y la Empresa. Obtenido de DialnetEIModeloDeLaTripleHeliceComoUnMedioParaLaVinculaci-3698520.pdf

Chiavenato, I. (2009). Gestión del talento humano. Mexico: Elsevier Editora Ltda.

Cubillos, N. (2017). Ocho de cada diez empresas capacitan a sus empleados. Obtenido de El Colombiano: http://www.elcolombiano.com/negocios/ocho-de-cada-diezempresas-capacitan-a-sus-empleados-YX7550256

DANE. (2014). Encuesta de Formación de Capital Humano.

Fundación COTEC. (2001). Innovación Tecnológica: Ideas basicas. Obtenido de http://www.uca.es/recursos/doc/Unidades/consejo_social/1801800_10320101035 32.pdf

Gonzalez, S. (s.f.). La Innovacion como fuente de Desarrollo.

Orozco, A. (2017). El Impacto de la Capacitacion. Mexico: UNID Editorial Digital.

Portafolio. (2014). El 80\% de las empresas tiene programas de capacitación. Obtenido de Portafolio: https://www.portafolio.co/economia/finanzas/80-empresas-programascapacitacion- 45304 
Revista Virtual Universidad Catolica del Norte . (2011). La capacitación a través de algunas teorías de aprendizaje y su influencia en la gestión de la empresa. Obtenido de https://www.redalyc.org/pdf/1942/194218961006.pdf

SENA. (2017). Dirección de planeación y direccionamiento corporativo. Obtenido de http://www.sena.edu.co/esco/transparencia/Lists/Informe\%20Estad\%C3\%ADstico/infoEstadisticoFeb2016.pdf

SENA. (2017). SENA presentó los resultados de su Gestión 2016 - 2017. Obtenido de http://www.sena.edu.co/es-co/Noticias/Paginas/noticia.aspx?IdNoticia=3245

Universidad Nacional de Quilmes. (1996). La innovación tecnológica: definiciones y elementos de base. Obtenido de Universidad Nacional de Quilmes: http://www.redalyc.org/articulo.oa?id=90711287005 
Anexo

\section{Diario de Campo:}

\section{Viernes 09 Noviembre}

Iniciamos nuestra agenda en México

D.F con la primera visita empresarial que se realizó en la Empresa Yakult, la cual es una compañía dedicada a la fabricación de productos lácteos basados en probióticos.

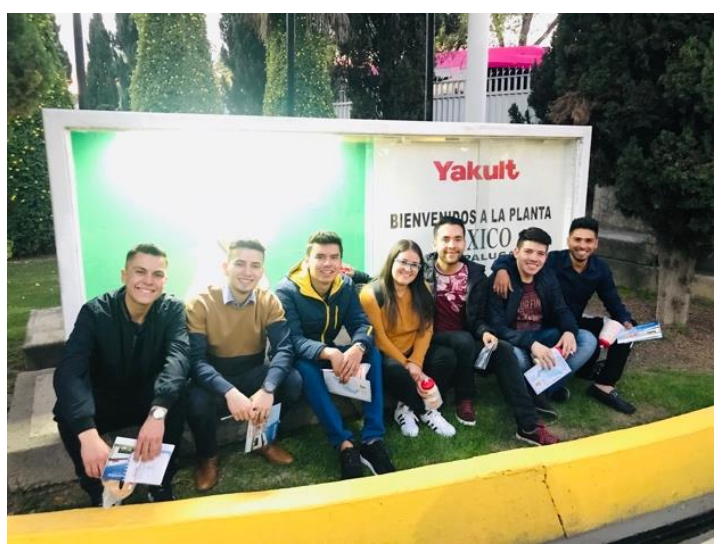

\section{Sábado 10 de Noviembre}

Iniciamos el día visitando el reconocido Tecnológico de Monterrey donde hicimos un recorrido por todo el campus con el fin de conocer las instalaciones del lugar, después del recorrido fuimos parte de un seminario de Emprendimiento, en el cual nos explicaron conceptos generales del emprendimiento y algunos Tips o consejos para empezar a emprender, de igual manera estuvimos presentes en la ponencia de unos estudiantes del Politécnico GranColombiano, donde pudimos evidenciar las diferentes ponencias de los postulados a la opción de grado.

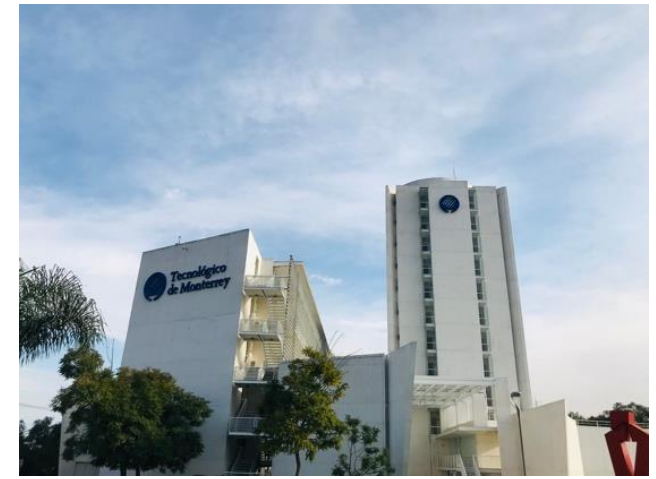

\section{Domingo 11 Noviembre}

Al comienzo del día visitamos sitios turísticos de México, como el Ángel de la independencia, La Basílica de la virgen de Guadalupe y terminamos en las Pirámides del Sol y La Luna, donde pudimos evidenciar un gran cantidad de interculturalización y conocer un poco de las tradiciones Mexicanas.

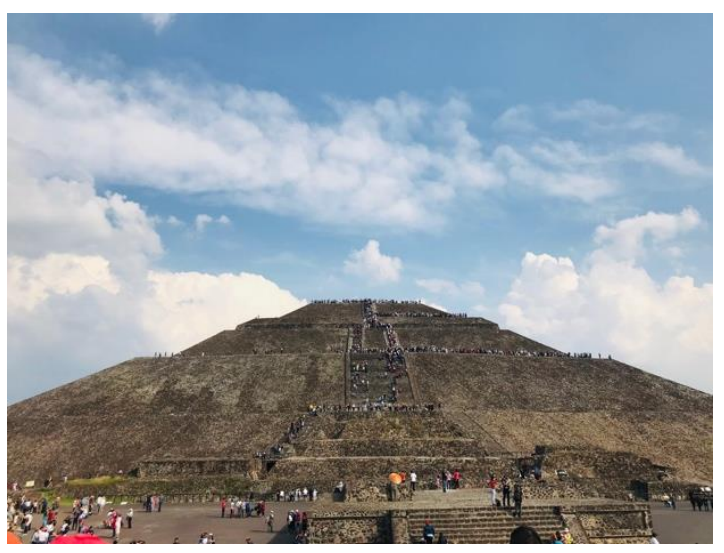

\section{Lunes 12 Noviembre}

En la noche anterior viajamos hacia Puebla para estar preparados para iniciar el itinerario del este día, comenzamos visitando Ternium que es una empresa industrial encargada de la 
producción de Alambrones, donde pudimos evidenciar todo el proceso de producción y la manera de cómo se supervisa dicho proceso, hay que recalcar que las condiciones de la empresa en cuanto a seguridad y salud en el trabajo son excelentes, preocupándose por el bienestar de cada personas que entra a la empresa.

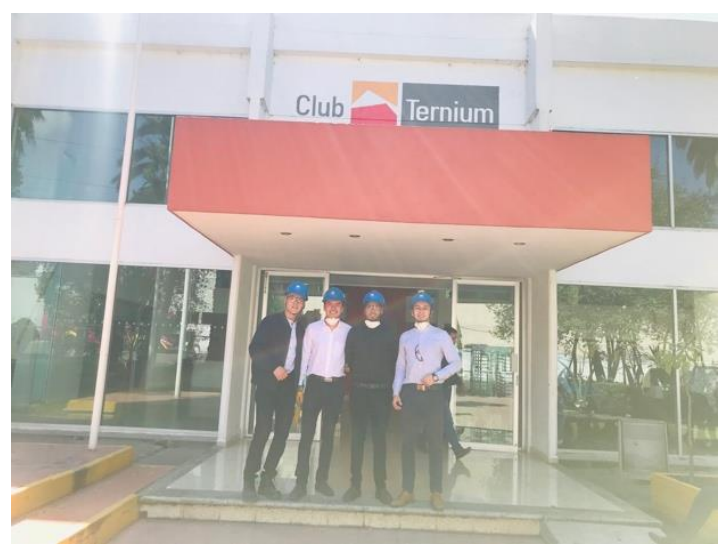

Después de conocer el proceso productivo de Ternium, nos dirigimos hacia Bimbo donde conocimos todo el proceso de producción de cada Línea de producto de esta empresa, conocimos avances tecnológicos implementados por Bimbo y la manera en cómo se optimizan los procesos mediante la adquisición e innovación de la maquinaria.

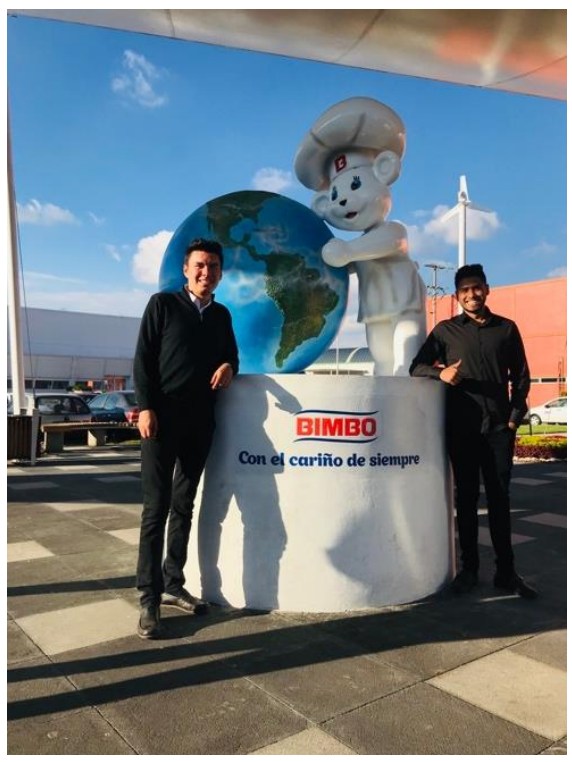

\section{Martes 13 Noviembre}

Comenzamos el día dirigiéndonos hacia el Centro de Especialización de Audi México ubicado en San José Chiapa, donde evidenciamos todo el proceso de capacitación de calidad de esta reconocida empresa, donde de igual manera nos informaron acerca de cómo realizan sus procesos de formación y como generan sus alianzas con el Gobierno y entidades educativas como la Universidad Tecnológica de Puebla.

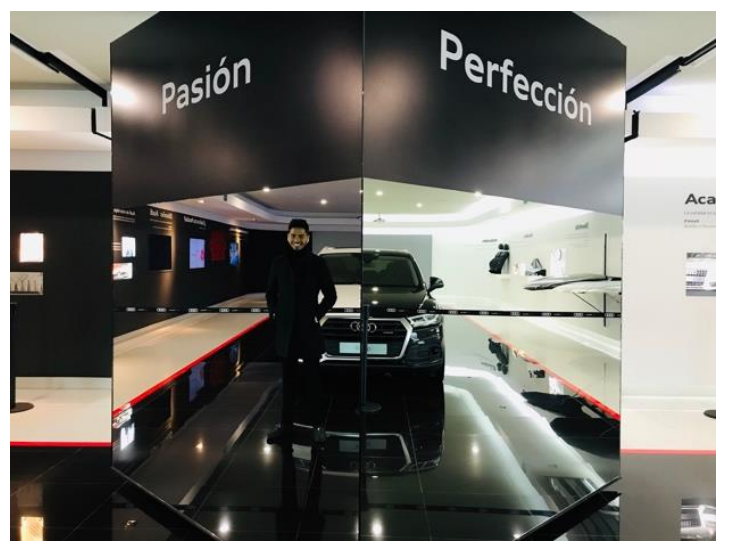




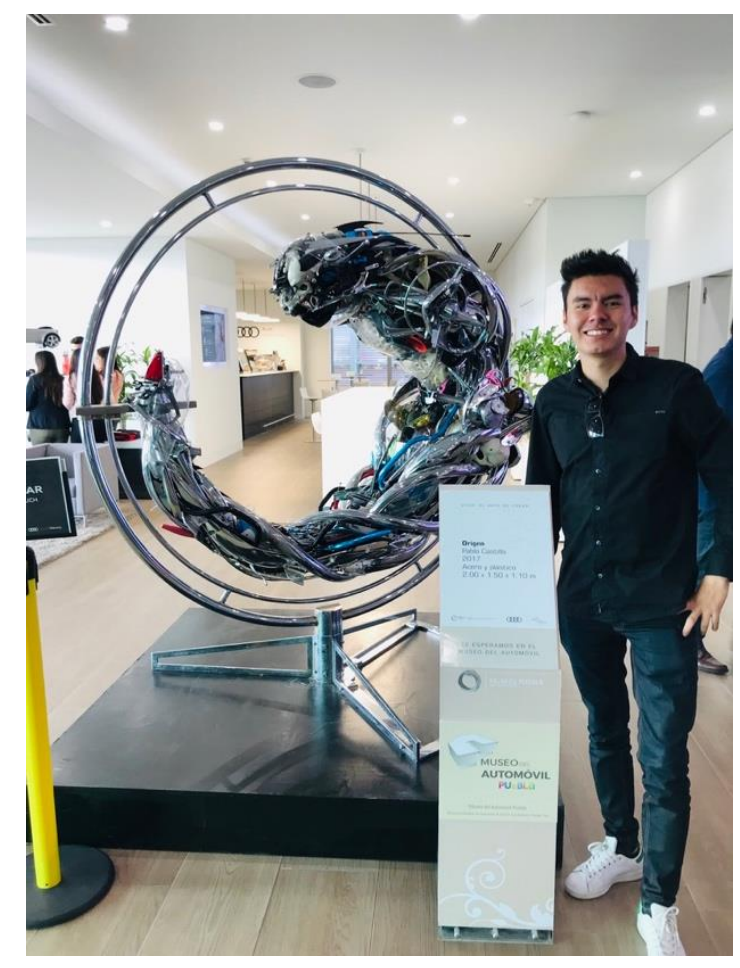

Después de visitar Audi México, nos dirigimos hacia Ingenio La Gloria que es una empresa encargada de la producción de productos derivados de la caña de azúcar, donde pudimos evidenciar toda la planta y maquinarias empeladas para llevar a cabo el proceso de los derivados de la caña de Azúcar.

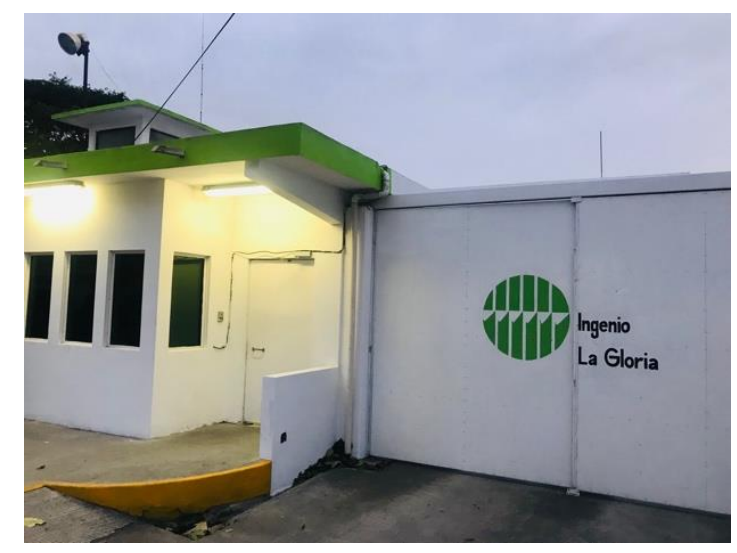

\section{Miércoles 14 Noviembre}

Iniciamos el itinerario de este día visitando la Universidad Veracruzana, donde recibimos una cordial bienvenida y estuvimos presentes en diferentes charlas acerca de Conceptos Generales de

La Administración, de igual manera realizamos el debido recorrido por la Universidad para conocer todas las áreas y salones.

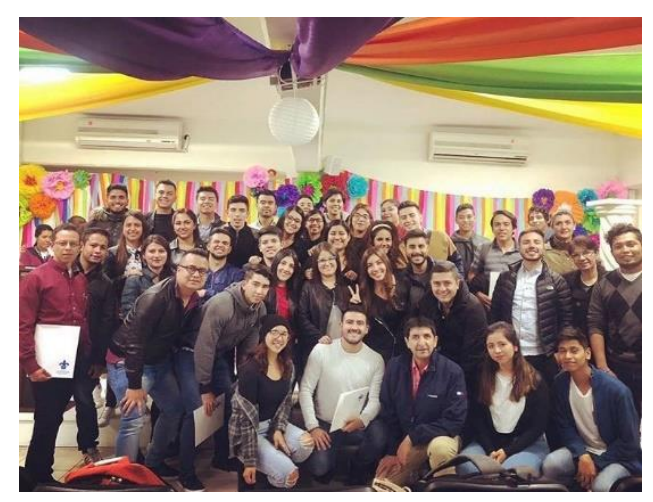

Terminamos nuestra migración visitando Quintana Roo, Cancún donde visitamos el Ministerio de Turismo donde nos contaron todos los planes que se tienen preparados para Quintana Roo en cuanto al tema de Turismo y de igual manera pudimos experimentar un tema claro de Cultura, interactuando con muchas personas de diferentes países, es ahí donde pudimos experimentar el inglés de cada uno de nosotros. 